\title{
Compact hybrid TM-pass polarizer for silicon-on-insulator platform
}

\author{
Muhammad Alam, ${ }^{*}$ J. Stewart Aitchsion, and Mohammad Mojahedi \\ Department of Electrical and Computer Engineering, University of Toronto, Toronto, Ontario M5S3G4, Canada \\ *Corresponding author: malam@ waves.utoronto.ca
}

Received 24 November 2010; revised 22 March 2011; accepted 26 March 2011; posted 28 March 2011 (Doc. ID 138734); published 18 May 2011

\begin{abstract}
Hybrid waveguides consisting of a metal plane separated from a high-index medium by a low-index spacer have recently attracted a lot of interest. TM and TE modes are guided in two different layers in these structures and their properties can be controlled in different manners by changing the waveguide dimensions and material properties. We examine the effects of different parameters on the characteristics of the two modes in such structures. We show that by properly choosing the dimensions, it is possible to cut off the TE mode while the TM mode can still be guided in a well-confined manner. Using this property of the hybrid guide, we propose a TM-pass polarizer. The proposed device is very compact and compatible with the silicon-on-insulator platform. Finite-difference time-domain simulation indicates that such a polarizer can provide a high extinction of the TE mode for a reasonable insertion loss of the TM mode. (C) 2011 Optical Society of America

OCIS codes: $\quad 130.3120,240.6690,260.3910,310.6628$.
\end{abstract}

\section{Introduction}

Surface plasmon (SP)—a hybrid surface wave that results from the coupling of an electromagnetic wave and free electrons in a metal-has attracted a lot of interest in recent years for its unique properties and its great potential for practical applications. For example, SP can offer subdiffraction-limited confinement of light not possible by other means [1]. Moreover, the presence of metal as a part of the guiding structure and the high field confinement on the metal surface make SP very attractive for biosensing [2] and electro-optic applications [3]. However, the application of SP is restricted by its large propagation loss. In the optical regime, metals are highly lossy and SP is strongly affected by this loss. One possible way to overcome this problem is to compensate for the loss by using an optical gain medium $[4,5]$. Another possibility is to integrate plasmonics with other photonic technologies, for example, with silicon-on-insulator (SOI). In this approach, the low-loss SOI guides will be used to guide light over large dis-

0003-6935/11/152294-05\$15.00/0

(C) 2011 Optical Society of America tances on an optical chip and plasmonic devices will be used locally to execute specific functions. One such function can be polarization control on an SOI integrated optical circuit. Given the fact that the high dielectric contrast of SOI waveguides makes them highly polarization-dependent, good control of the polarization state is necessary for proper operation of SOI-based integrated optical circuits. This can be achieved by using a polarizer to extinguish the unwanted polarization state. Although numerous polarizer designs are available in the literature, there are relatively few polarizer designs that are compact and compatible with SOI technology $[\underline{6}, 7]$. To implement the plasmonic waveguide-based polarizer and other devices for SOI, a plasmonic guide is needed that is fully compatible with SOI fabrication technology. The guide dimensions should be comparable with SOI guides so that both technologies can be integrated easily. The selected plasmonic guide should also provide a good compromise between loss and confinement. All these properties can be satisfied by our (along with others) recently proposed hybrid plasmonic waveguide [8-12]. Here, we present a SOI compatible TM-pass polarizer using the hybrid guiding scheme. 

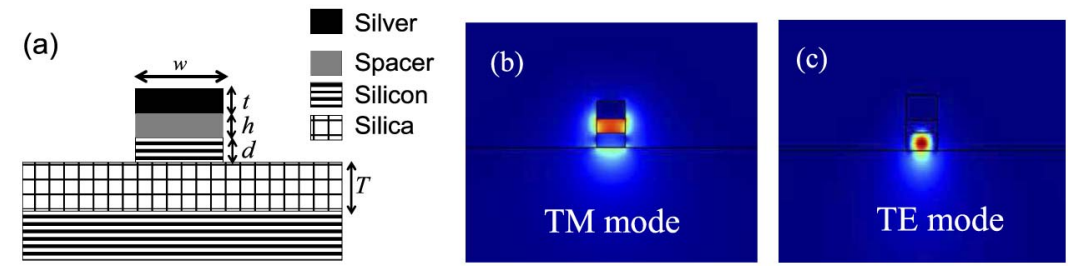

Fig. 1. (Color online) (a) Cross section of the hybrid waveguide. (b) Electric field intensity for TM mode. (c) Electric field intensity for TE mode. Waveguide dimensions are $w=350 \mathrm{~nm}, t=200 \mathrm{~nm}, h=150 \mathrm{~nm}, d=150 \mathrm{~nm}, T=2 \mu \mathrm{m}$. Wavelength of operation is $1550 \mathrm{~nm}$ and the spacer is silica.

\section{Proposed TM Polarizer}

Figure 1(a) shows the hybrid guide chosen in this work [12]. It consists of a silver layer of dimension $w \times t$ separated from a high-index layer (silicon) of dimension $w \times d$ by a low-index spacer of dimension $w \times h$. Electric field intensity profiles for the TM and TE modes, obtained by finite element code Comsol Multiphysics, are shown in Figs. 1(b) and 1(c), respectively. Material properties for silica and silicon are taken from [13] and that of silver is from [14]. The TM mode supported by the hybrid guide is a supermode resulting from the coupling between the SP mode guided by the silver-silica interface and the dielectric waveguide mode guided by the silicon layer. The supermode is highly concentrated in the low-index spacer region (silica, in the present case). On the other hand, the TE mode is very similar to conventional dielectric waveguide mode and is concentrated in the high-index region, i.e., in the silicon layer.

Since the two modes are guided in two different layers, changing the dimensions and material properties of these layers will affect the two modes in different ways. For designing practical devices using the hybrid guide, it is necessary to have a clear understanding of how the choice of waveguide dimensions and material properties affect the mode characteristics. Therefore, we have carried out a detailed numerical analysis to investigate these effects. Some of these results are presented in Figs. 2-4. Figure $\underline{2}$ shows the effects of spacer permittivity $\varepsilon_{\text {spacer }}$ on the effective mode index $N_{\text {eff }}$ and the propagation distance of the TM mode. Here, $N_{\text {eff }}$ is defined as $N_{\text {eff }}=k / k_{0}$ where $k$ is the real part of the

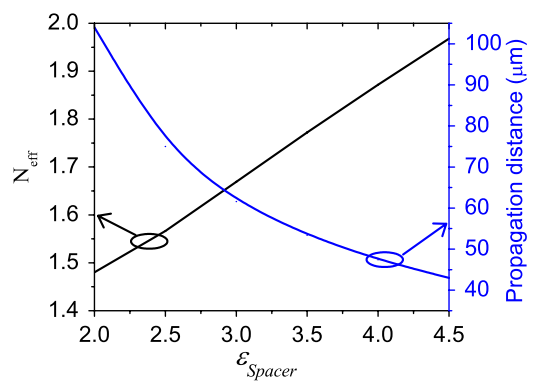

Fig. 2. (Color online) Effect of varying spacer permittivity $\left(\varepsilon_{\text {spacer }}\right)$ on effective mode index $\left(N_{\text {eff }}\right)$ and propagation distance for the TM mode. Waveguide dimensions are $w=350 \mathrm{~nm}, t=200 \mathrm{~nm}$, $h=100 \mathrm{~nm}, d=100 \mathrm{~nm}$ and $T=2 \mu \mathrm{m}$. propagation constant and $k_{0}=\omega / c$ is the free space wave number. The propagation distance is defined as the distance over which guided power drops to 1/e of its initial magnitude. Increase of spacer permittivity results in increase of $N_{\text {eff }}$ and decrease of propagation distance. Two possible choices of spacer materials are silica and silicon nitride, which have permittivity 2.08 and 4 , respectively. In this work, we have chosen silica as the spacer material to ensure low loss in the hybrid section.

Figures 3(a) and 3(b) show the effects of spacer thickness $(\overline{h)}$ and silicon thicknesses $(d)$ on effective mode index $\left(N_{\text {eff }}\right)$ for TM and TE modes, respectively. Reduction of spacer thickness results in an increase of $N_{\text {eff }}$ for the TM mode, but the opposite is true for the TE mode. When silicon thickness is large (e.g., $d=200 \mathrm{~nm}$ ), both modes exist for any spacer thickness considered here. For small silicon thickness (e.g., $d=100 \mathrm{~nm}$ ), the hybrid waveguide supports only the TM mode.

Figure 4 shows the variations of propagation distance for the two modes with varying waveguide dimensions. The TM mode is lossier than the TE mode for all choices of silicon and spacer thicknesses considered in this work. The propagation distance of the TM mode decreases monotonically with reduced spacer thickness. Propagation distance of the TE mode follows the same trend when silicon thickness is large (e.g., $d=200 \mathrm{~nm}$ ). For a reduced silicon thickness (e.g., $d=150 \mathrm{~nm}$ ), the propagation distance of the TE mode is small for intermediate values of spacer thickness.

To understand the reason behind the variations of mode characteristics presented in Figs. 3 and 4 , we have examined the field profiles of both modes. Figure 5 shows the electric field intensity of the TM mode for different spacer thicknesses $(h)$. The dominant electric field component of the TM mode is perpendicular to the metal, as shown by the electric field arrows in the figures. With reduced spacer thickness, the mode is more tightly confined and $N_{\text {eff }}$, is larger as shown in Fig. 3(a). Like all plasmonic guides, this increased confinement is accompanied by reduced propagation distance, as shown in Fig. 4(a).

Figure 6 shows the electric field intensity of the TE mode for a number of spacer thicknesses. The field is concentrated in the high-index region (silicon), and since the silicon film is thin in this case $(d=150 \mathrm{~nm})$, the field is loosely confined. For large spacer thickness, the mode is not significantly affected by the 

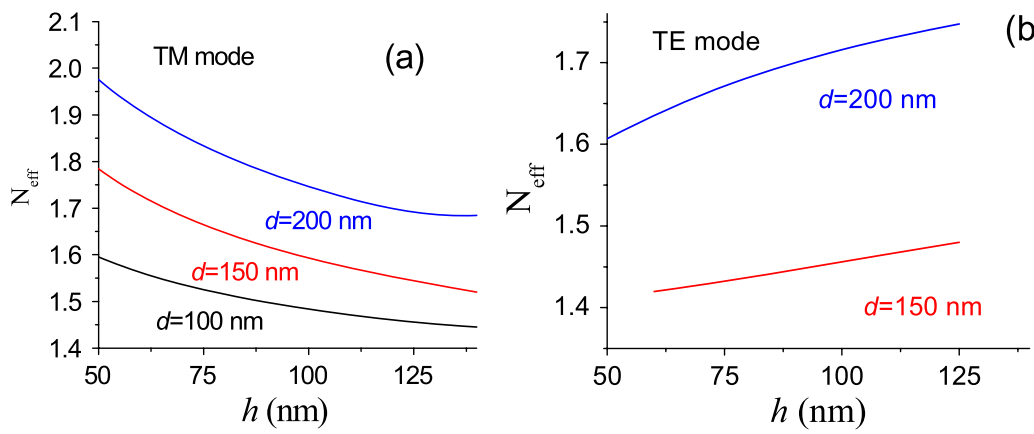

Fig. 3. (Color online) (a) and (b): Variations of effective mode index $\left(N_{\text {eff }}\right)$ for TM and TE modes with spacer thickness $(h)$ for a number of fixed silicon thicknesses $(d)$. Other dimensions are $w=350 \mathrm{~nm}, t=200 \mathrm{~nm}, T=2 \mu \mathrm{m}$. Wavelength of operation is $1.55 \mu \mathrm{m}$.

metal and propagation distance is large (propagation loss is low). As the spacer thickness is reduced, the mode is more affected by the presence of metal, and propagation distance decreases (propagation loss increases). According to the boundary conditions of the electric field, the tangential electric field is zero on the surface of a perfect conductor. Though silver is not a perfect conductor in near-IR, it still has a large negative real part of permittivity and can be considered a "good conductor." Hence, for a small spacer thickness, the dominant electric field component of the TE mode, which is tangential to the metal surface, is pushed away from the metal. The net result is the following: for small spacer and silicon thicknesses, the TE mode is pushed into the substrate and approaches the cutoff condition, as shown in Fig. 6(c). Since in this case, the field is mostly in the substrate (a "good dielectric"), metallic losses are reduced. Therefore, for a thin silicon film (e.g., $d=150 \mathrm{~nm}$ ), the propagation distance is minimized for an intermediate choice of spacer thickness as shown in Fig. $\underline{4(b)}$.

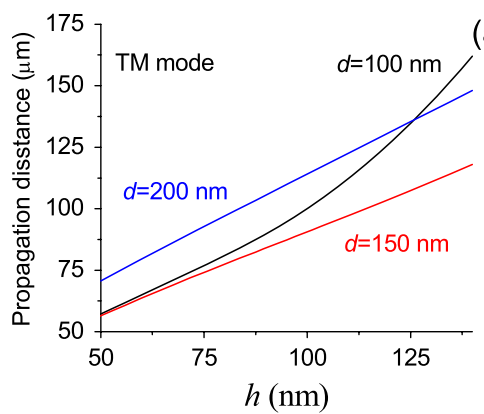

(a)

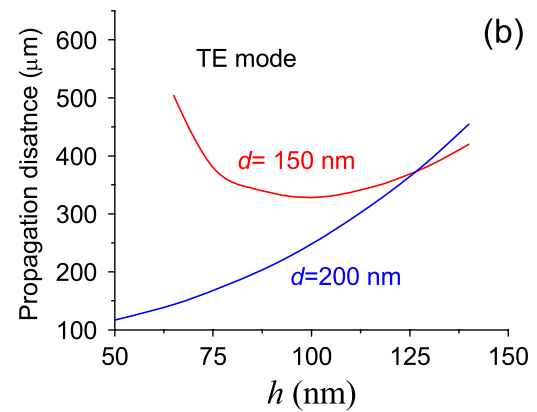

Fig. 4. (Color online) (a) and (b): Variations of propagation distance for TM and TE modes with spacer thickness $(h)$ for a number of fixed silicon thicknesses $(d)$. Other dimensions are $w=350 \mathrm{~nm}, t=200 \mathrm{~nm}, T=2 \mu \mathrm{m}$. Wavelength of operation is $1.55 \mu \mathrm{m}$.
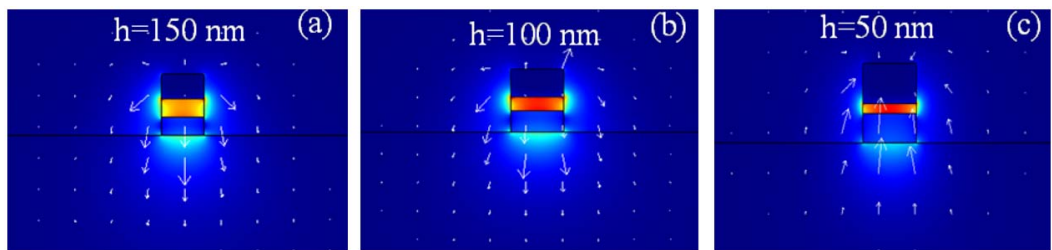

Fig. 5. (Color online) Variations of electric field intensity for the TM mode with spacer thickness $h$. (a) $h=150 \mathrm{~nm}$, (b) $h=100 \mathrm{~nm}$, (c) $h=50 \mathrm{~nm}$. The other dimensions are $w=350 \mathrm{~nm}, t=200 \mathrm{~nm}, d=150 \mathrm{~nm}, T=2 \mu \mathrm{m}$. Wavelength of operation is $1.55 \mu \mathrm{m}$. 

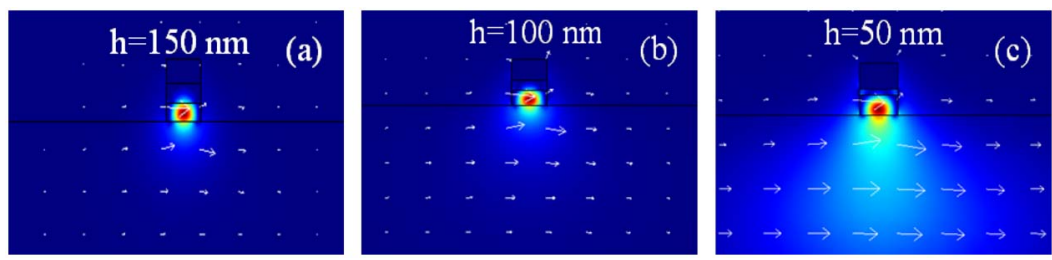

Fig. 6. (Color online) Variations of electric field intensity for the TE mode with spacer thickness $h$. (a) $h=150 \mathrm{~nm}$, (b) $h=100 \mathrm{~nm}$, (c) $h=50 \mathrm{~nm}$. The other dimensions are $w=350 \mathrm{~nm}, t=200 \mathrm{~nm}, d=150 \mathrm{~nm}, T=2 \mu \mathrm{m}$. Wavelength of operation is $1.55 \mu \mathrm{m}$.
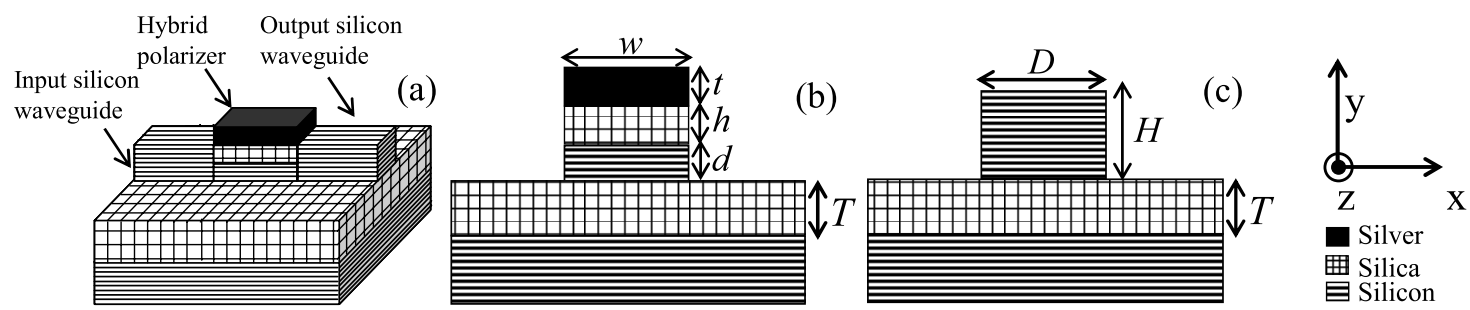

Fig. 7. (a) Three-dimensional schematic of the complete TM polarizer. (b) Cross section of the hybrid section. (c) Cross section of the input and output silicon waveguides. The coordinate system is defined with respect to (b) and (c) with the $x z$ plane coinciding with the silicon waveguide-buried oxide interface and its origin located at the beginning of the hybrid waveguide section.

perfectly matched layers (PML). Simulations were carried out on multiple processors in parallel on the high-performance computer cluster Westgrid. Details of the simulation process can be found in [15]. Figures 8(a) and 8(b) show the electric field intensity profiles of the TM and TE modes, respectively, along the polarizer for $1.55 \mu \mathrm{m}$ wavlength for an $18-\mu \mathrm{m}$-long polarizer. The hybrid polarizer section is located between $z=0 \mu \mathrm{m}$ and $z=18 \mu \mathrm{m}$. The TM mode is well guided from the input to the output, although it suffers some propagation losses. The TE mode, on the other hand, is below cutoff, and hence is unable to propagate through the hybrid section.

In Fig. 9 , we have plotted the variations of insertion losses of the TM and TE modes with polarizer length for two different values of $T$. The extinction ratio (difference between the insertion losses of TM and TE modes) is high for both cases. Insertion loss of the TM mode, however, is not negligible, and for large values of $T$, the polarizer described in [6] outperforms our hybrid polarizer in terms of both $\mathrm{TM}$ insertion loss and extinction ratio. However, there are
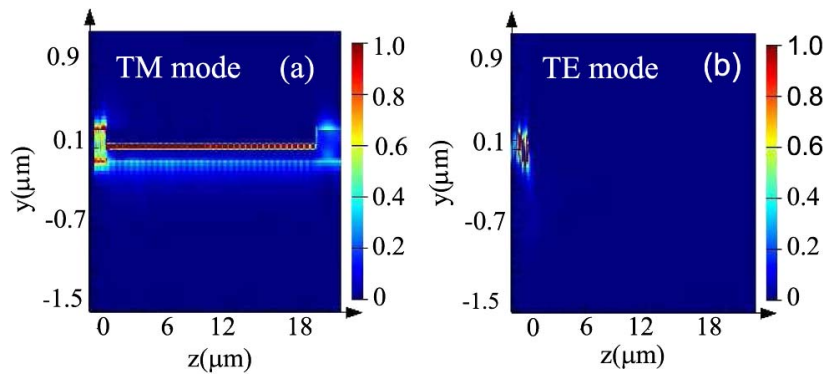

Fig. 8. (Color online) Electric field intensity plot for the light propagating through the polarizer. (a) TM mode, (b) TE mode for the TM-pass polarizer. Device dimensions are $w=350 \mathrm{~nm}, t=$ $200 \mathrm{~nm}, h=50 \mathrm{~nm}, d=100 \mathrm{~nm}, T=1 \mu \mathrm{m}$. Dimensions of input and output waveguides are $D=350 \mathrm{~nm}$ and $H=300 \mathrm{~nm}$. Spacer is silica. Wavelength of operation is $1.55 \mu \mathrm{m}$. practical applications, such as optical amplifiers implemented on a SOI substrate, where a thin oxide layer is required [16]. For $T=1 \mu \mathrm{m}$, the extinction ratio for our $18-\mu \mathrm{m}$-long hybrid polarizer is $21 \mathrm{~dB}$, compared to $14 \mathrm{~dB}$ for the polarizer described in [6]. Therefore, for a thin oxide substrate, the use of the hybrid polarizer is more advantageous than the polarizer described in [6]. The hybrid polarizer can also provide a high extinction ratio for a shorter polarizer length. For example, a 13- $\mu$ m-long hybrid polarizer (with all other dimensions the same as in Fig. 9 caption) provides $21.8 \mathrm{~dB}$ and $3.2 \mathrm{~dB}$ insertion loss for the TM and TE modes, respectively, i.e., an extinction ratio of $18.6 \mathrm{~dB}$. If $(h)$ is increased from $50 \mathrm{~nm}$ to $150 \mathrm{~nm}$, the TE mode is no longer far below cutoff, and hence the extinction ratio for a $18-\mu \mathrm{m}$-long polarizer drops from $21 \mathrm{~dB}$ to $7.7 \mathrm{~dB}$. Choosing a small value of $h$, therefore, is essential for proper operation of the polarizer.

The insertion loss of the TM mode is caused by three factors: material loss due to the presence of metal, mismatch of mode profile between the hybrid segment and input/output silicon waveguides, and reflection caused by the mode index mismatch between the hybrid guide and input/output waveguides. Less than one-third of the insertion loss results from material loss. The mode size in the hybrid waveguide is very similar to that in the silicon input/output waveguide, and hence losses resulting from mode-size mismatch are expected to be low. However, because of the difference in the effective mode indices for the TM modes in the hybrid section from those in the input/output silicon waveguide sections, significant reflection occurs. One solution to this is to use a taper section between the input/ output waveguides and the hybrid plasmonic section. Design of tapers for mode matching is a well-studied and understood topic, and hence will not be discussed in this work. 

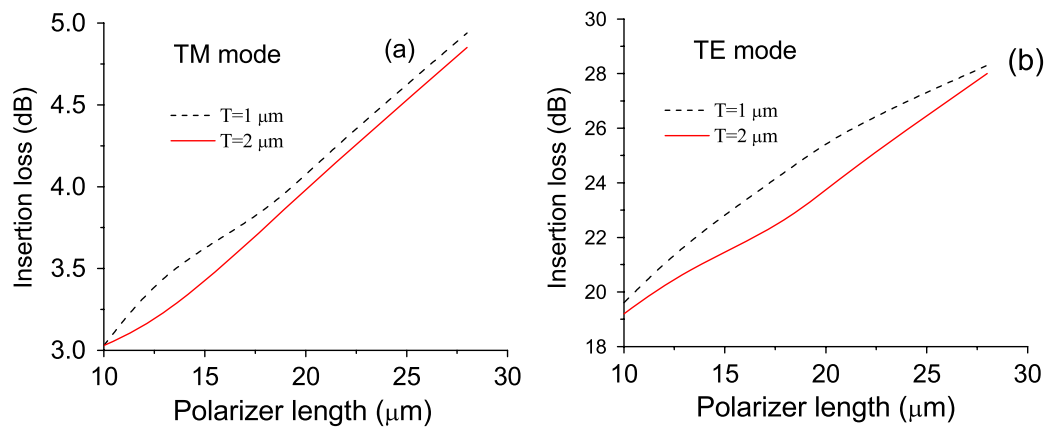

Fig. 9. (Color online) Insertion losses of the TM-pass polarizer for two different buried oxide thicknesses. (a) TM mode, (b) TE mode. Device dimensions are $w=350 \mathrm{~nm}, t=200 \mathrm{~nm}, h=50 \mathrm{~nm}, d=100 \mathrm{~nm}$. Spacer is silica. Dimensions of input and output waveguides are $D=350 \mathrm{~nm}$ and $H=300 \mathrm{~nm}$. Spacer is silica. Wavelength of operation is $1.55 \mu \mathrm{m}$.

\section{Conclusion}

We have proposed a compact hybrid TM-pass polarizer that is fully compatible with SOI technology. The effectiveness of the proposed polarizer is confirmed by FDTD simulations. To the best of our knowledge, the proposed device is the first application of the hybrid plasmonic waveguide as a polarizer for integrated optics. Many other hybrid devices, for example, TE-pass polarizer, polarization-independent directional coupler, and polarization rotator, can be implemented using this technology and will be reported in our future publications.

We would like to acknowledge the use of computing resources from WestGrid. This work was supported by the Natural Sciences and Engineering Research Council (NSERC) of Canada under grant no. 480586 and BiopSys Network under grant no. 486537.

\section{References}

1. W. L. Barnes, A. Dereux, and T. W. Ebbesen, "Surface plasmon subwavelength optics," Nature 424, 824-830 (2003).

2. J. Homola, "Present and future of surface plasmon resonance biosensors," Anal. Bioanal. Chem. 377, 528-539 (2003).

3. A. V. Krasavin and A. V. Zayats, "Electro-optic switching element for dielectric-loaded surface plasmon polariton waveguides," Appl. Phys. Lett. 97, 041107 (2010).

4. M. Z. Alam, J. Meier, J. S. Aitchison, and M. Mojahedi, "Gain assisted surface plasmon polariton in quantum wells structure," Opt. Express 15, 176-182 (2007).

5. I. D. Leon and P. Berini, "Amplification of long-range surface plasmons by a dipolar gain medium," Nat. Photon. 4, 382-387 (2010).
6. Q. Wang and S.-T. Ho, "Ultra compact TM-pass silicon nanophotonic waveguide polarizer and design," IEEE Photon. J. 2, 49-56 (2010).

7. Y. Cui, Q. Wu, E. Schonbrun, M. Tinker, J.-B. Lee, and W. Park, "Silicon-based 2-D slab photonic crystal TM polarizer at telecommunication wavelength," IEEE Photonics Technol. Lett. 20, 641-643 (2008).

8. M. Z. Alam, J. Meier, J. S. Aitchison, and M. Mijahedi, "Super mode propagation in low index medium," Conference on Lasers and Electro-Optics (CLEO) and Quantum Electronics and Laser Science Conference (QELS) (IEEE, 2010).

9. M. Z. Alam, J. Meier, J. S. Aitchison, and M. Mijahedi, "Propagation characteristics of hybrid modes supported by metallow-high index waveguides and bends," Opt. Express 18, 12971-12979 (2010).

10. M. Fujii, J. Leuthold, and W. Freude, "Dispersion relation and loss of sub-wavelength confined mode of metal-dielectric-gap optical waveguides," IEEE Photonics Technol. Lett. 21, 362-364 (2009).

11. R. F. Oulton, V. J. Sorger, D. A. Genov, D. F. P. Pile, and X. Zhang, "A hybrid plasmonic waveguide for subwavelength confinement and long-range propagation," Nat. Photon. 2, 496-500 (2008).

12. D. Dai and S. He, "A silicon-based hybrid plasmonic waveguide with a metal cap for a nano-scale light confinement," Opt. Express 17, 16646-16653 (2009).

13. E. D. Palik, Handbook of Optical Constants of Solids (Academic, 1985).

14. P. B. Johnson and R. W. Christy, "Optical constants of noble metals," Phys. Rev. B 6, 4370-4379 (1972).

15. FDTD Solutions Reference Guide (Lumerical Solutions 2009).

16. H. Park, A. W. Fang, O. Cohen, R. Jones, M. J. Paniccia, and J. E. Bowers, "A hybrid AlGaInAs-silicon evanescent amplifier," IEEE Photonics Technol. Lett. 19, 230-232 (2007). 